\title{
Structural Impact of Proline-Directed Pseudophosphorylation at AT8, AT100, and PHF1 Epitopes on 441-Residue Tau
}

\author{
Stefan Bibow, ${ }^{\dagger}$ Valéry Ozenne, ${ }^{\perp}$ Jacek Biernat, ${ }^{\S, \|}$ Martin Blackledge, ${ }^{\perp}$ Eckhard Mandelkow, ${ }^{\S, \| l}$ and \\ Markus Zweckstetter, ${ }^{*,+}$ \\ ${ }^{\dagger}$ Department of NMR-based Structural Biology, Max Planck Institute for Biophysical Chemistry, Am Fassberg 11, \\ 37077 Göttingen, Germany \\ ${ }^{\ddagger}$ DFG Center for the Molecular Physiology of the Brain, 37073 Göttingen, Germany \\ ${ }^{\S}$ Max Planck Unit for Structural Molecular Biology, c/o DESY, Notkestrasse 85, 22607 Hamburg, Germany \\ "DZNE, German Center for Neurodegenerative Diseases, c/o CAESAR, Ludwig-Erhard-Allee 2, 53175 Bonn, Germany \\ ${ }^{\perp}$ Institut de Biologie Structurale Jean-Pierre Ebel, CEA-CNRS-UJF UMR 5075, 41 Rue Jules Horowitz, Grenoble 38027, France
}

Supporting Information

ABSTRACT: The intrinsically disordered protein tau becomes excessively phosphorylated and aggregates into neurofibrillary tangles in Alzheimer's disease. To obtain insight into the structural consequences of phosphorylation, we characterized a mutant protein of tau in which epitopes recognized by Alzheimer diagnostic antibodies were mimicked by mutation to glutamic acid [AT8 (S199E, S202E, T205E), AT100 (T212E and S214E), and PHF1 (S396E and S404E)]. A large number of distance restraints obtained from NMR paramagnetic relaxation enhancement in combination with ensemble conformer calculations demonstrate that pseudophosphorylation causes an opening of the transient folding of tau. Together with previous studies on the Parkinson-related protein $\alpha$-synuclein, our data indicate that networks of transient long-range interactions are common properties of intrinsically disordered proteins and that their modulation is important for aggregation.

\footnotetext{
A ggregation of the microtubule-associated protein tau into Aneurofibrillary tangles is the pathological hallmark of a variety of dementias. ${ }^{1,2}$ For reasons not yet known, tau becomes excessively phosphorylated in Alzheimer's brains and as a result no longer binds properly to microtubules. The unbound tau is free to undergo abnormal aggregation. In vivo, hyperphosphorylation of tau precedes tangle formation. ${ }^{3}$ At least 30 phosphorylation sites in tau filaments have been identified. ${ }^{4}$ Phosphorylation at serine/proline and threonine/proline motifs in the flanking regions of the repeat domain of tau has only a moderate influence on tau-microtubule interactions but is upregulated in Alzheimer's disease. ${ }^{5}$

Tau is a prototypical intrinsically disordered protein that does not assume a rigid tertiary or secondary structure but populates an ensemble of interconverting structures in solution. ${ }^{6,7}$ Because of the inherent flexibility of tau, NMR spectroscopy is the only method that allows a description of its conformations and dynamics with high resolution. ${ }^{8}$ We have recently shown that it is possible to obtain the complete backbone resonance assignment of the longest isoform of human tau and demonstrated that 441-residue tau has a distinct domain character with an intricate network of long-range interactions. ${ }^{7}$
}

Here we investigate changes in the local and global structure of 441-residue tau related to phosphorylation in the epitopes recognized by the Alzheimer diagnostic antibodies AT8 (S199E, S202E, T205E), AT100 (T212E and S214E), and PHF1 (S396E and S404E). To avoid the ambiguities of heterogeneous phosphorylation, we cloned "pseudophosphorylation" mutants of tau in which serine and threonine residues were converted into glutamic acid. The same mutant protein was previously shown to aggregate slightly faster in comparison with wild-type (wt) tau. ${ }^{9}$ In addition, a different six-site pseudophosphorylation mutant (S199E, S202E, T205E, T231E, S396E, and S404E) had a decreased rate of elongation and a pronounced lag time of aggregation. ${ }^{10}$ We determined cross-validated ensembles of wild-type (wt) and pseudophosphorylated tau using a large number of long-range distance restraints. Our data reveal that pseudophosphorylation weakens the transient folding of tau.

To obtain insight into transient long-range interactions in wt and pseudophosphorylated (so-called E-mutant) tau, we used the technique of paramagnetic relaxation enhancement (PRE). ${ }^{11}$ Covalent attachment of a spin label to a cysteine induces PRE of the NMR signals of the protein within a distance of $\sim 25 \AA$. The relaxation enhancement can then be quantified by comparison of NMR signal intensities in two-dimensional correlation experiments recorded in the paramagnetic $\left(I_{\text {para }}\right)$ and diamagnetic $\left(I_{\text {dia }}\right)$ states. An $I_{\text {para }} / I_{\text {dia }}$ intensity ratio of $>0.95$ indicates a distance exceeding $25 \AA$. We attached the paramagnetic nitroxide label MTSL to 10 different positions (C15, C72, C125, C178, C239, C256, C322, C352, C384, and C416) uniformly distributed along the primary sequence of wt and E-mutant 441-residue tau (Figures 1 and 2). In addition, PRE measurements were performed in which a spin label was attached simultaneously to the two native cysteines C291 and C322 of tau. Our previous characterization of wt tau was based on six MTSL positions.

In Figure 1, a comparison between the PRE broadening profiles of the amide protons of wt and E-mutant tau is shown for MTSL attached at positions 239 and 384, which are close to the sites of pseudophosphorylation. In agreement with our previous study, ${ }^{7}$ the spin label at position 239 strongly attenuated

Received: June 23, 2011

Published: September 12, 2011 
the signals of 60 residues at the $\mathrm{N}$-terminus with weaker broadening extending up to residue 140 . In addition, weak paramagnetic broadening was observed for about 60 residues upstream and downstream of the site of the spin label (Figure 1a). Very

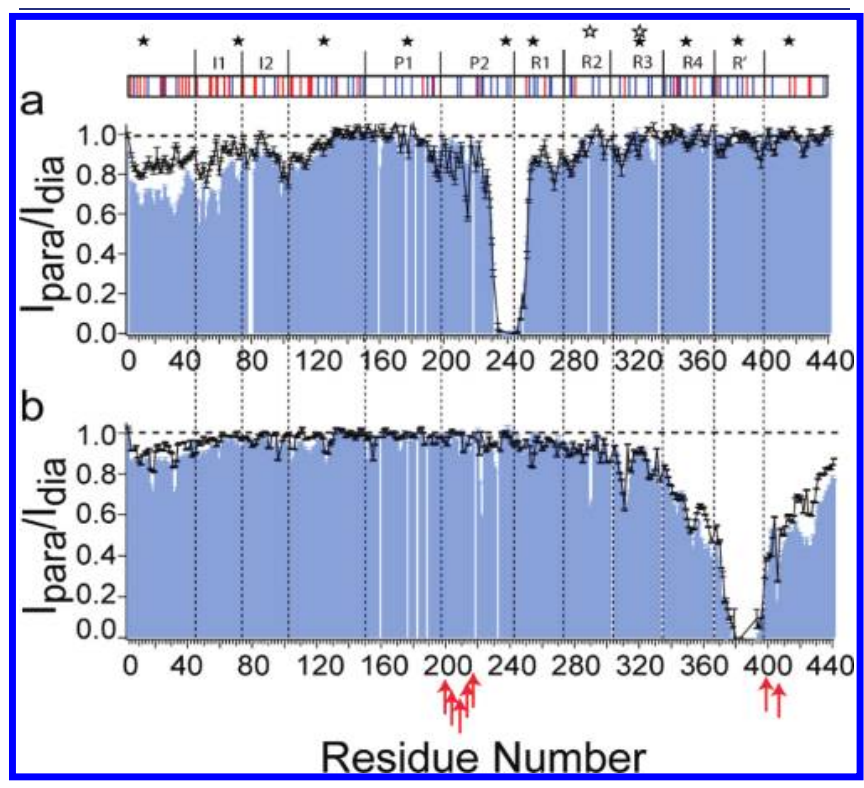

Figure 1. PRE broadening profiles of amide protons of wt (blue) and E-mutant 441-residue tau (black line) with MTSL attached to positions (a) 239 and (b) 384. Arrows mark sites of mutation to glutamic acid. Intensity ratios were averaged over a three-residue window. Decreases in peak intensity ratios that occur far from the site of spin-labeling $(>10$ residues) are indicative of long-range contacts. The domain organization shown at the top (inserts I1, I2; proline-rich regions P1, P2; domain of repeats $\mathrm{R} 1-\mathrm{R} 4$; pseudorepeat $\mathrm{R}^{\prime}$ ) highlights the location of negative (red) and positive (blue) charges. The sites of spin labeling are indicated by solid (single spin label) and open (double spin label) stars. similar PRE profiles were obtained when the diamagnetic state was obtained by addition of ascorbic acid instead of dithiothreitol (DTT) (Figure S1 in the Supporting Information). For residues 150-441 of the E-mutant, a highly similar PRE profile was observed. However, at the N-terminus of the E-mutant, the PRE intensity ratios were higher than in wt tau by up to $34 \%$ for residues M31-D34. The reduced paramagnetic effect demonstrates that pseudophosphorylation in the regions flanking the repeat domain of tau attenuate their transient interaction with the N-terminal domain. MTSL attached at position 384 induced a broad PRE profile around the attachment site and weak paramagnetic enhancement at the $\mathrm{N}$-terminus for both proteins. In the E-mutant, the paramagnetic effect was reduced for residues 410-441 (Figure 1b).

The PRE profiles in Figure 1 provide information only about changes in transient long-range structure involving residues 239 and 384. To probe the effect of pseudophosphorylation on the ensemble of conformations in other regions of tau, nine additional PRE profiles were compared (Figure S2). The NMR data demonstrate that residue stretches harboring each of the nine sites are involved in long-range interactions in both wt and E-mutant tau. For all attachment sites, the paramagnetic effect was at least slightly reduced in distinct regions upon pseudophosphorylation. According to the PRE profile for MTSL at position 15 , pseudophosphorylation in the regions flanking the repeat domain causes a weakening of the long-range contact between the $\mathrm{N}$ - and C-termini (Figure S2). A weakened contact between the two termini is in agreement with a lower fluorescence resonance energy transfer (FRET) efficiency between residues 432 and 17 in the E-mutant relative to wt tau. ${ }^{9}$ However, the FRET efficiencies between residues 310 and 17 and between residues 432 and 322 were larger in the E-mutant than in the wt protein, ${ }^{9}$ in apparent contrast to the NMR PRE profiles. We attribute the differences to the use of two hydrophobic labels (tryptophan and dansyl group) in the FRET studies.

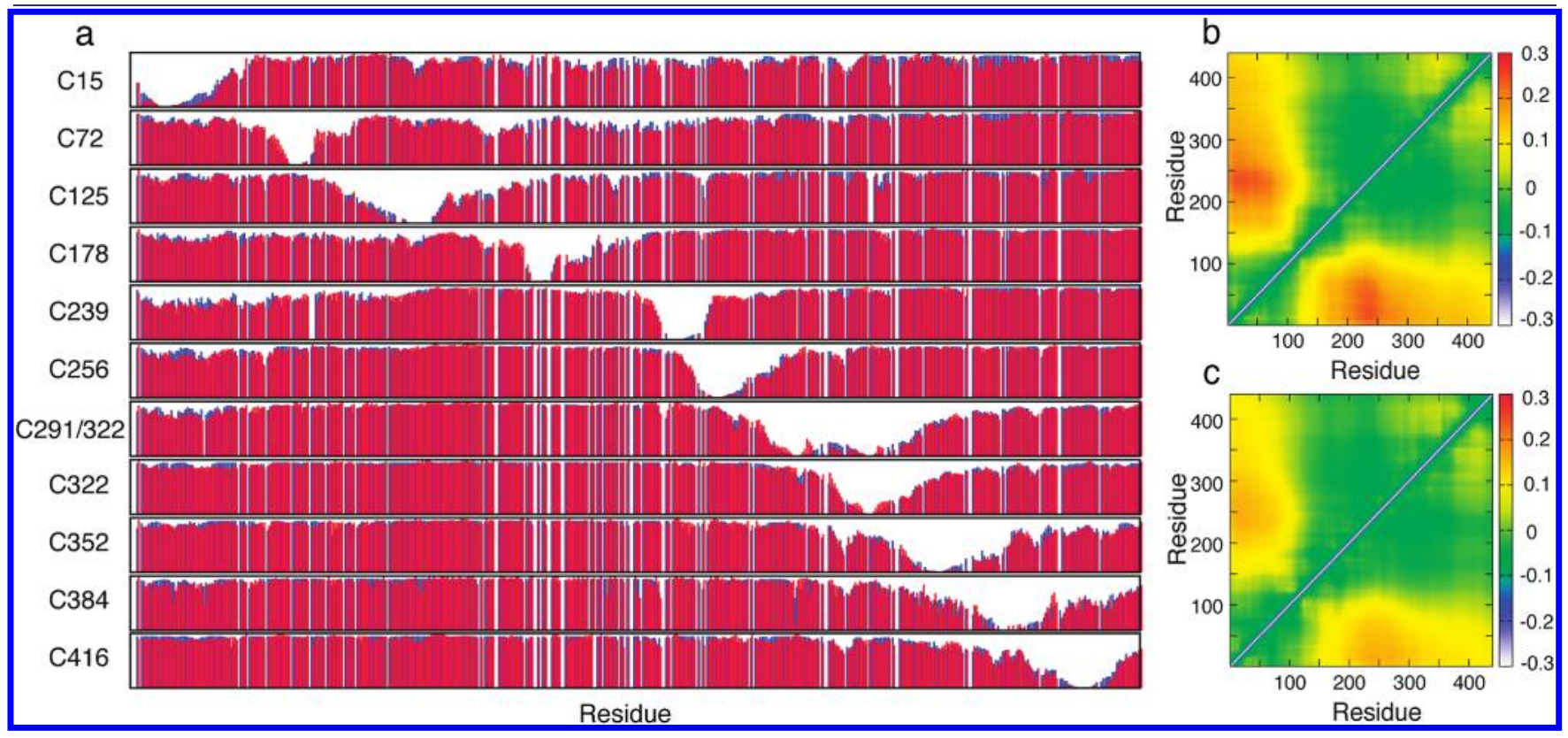

Figure 2. PREs and long-range contacts in the representative ensembles of ( $a, b)$ wt and (c) E-mutant 441-residue tau. (a) Comparison of experimental PREs (red) and values back-calculated from the representative ensemble of wt tau (blue). ( b, c) Contacts are plotted as $\log \left(d_{i j} / d_{i j, \text { ref }}\right)$, where $d_{i j, \text { ref }}$ refers to the distance in the reference ensemble of 27000 structures and $d_{i j}$ refers to the distance in the selected ensemble [see Figure S14 for a difference plot of (b) and (c)]. 
A hint concerning the mechanism of the pseudophosphorylation-induced weakening of transient long-range interactions was provided by an analysis of the charge distribution of tau. While the central region of tau is predominantly positively charged, an excess of negative charge was found for the $120 \mathrm{~N}$-terminal residues as well as at the $\mathrm{C}$-terminus. Introduction of five glutamic acid residues in the region 199-214 changes the net charge in the proline-rich region P2 from +2 to -3 (Figure 1a). The altered electrostatic properties result in a reduced Coulombic attraction between P2 and the positively charged $\mathrm{N}$-terminal domain.

The overall dimensions of the ensemble of conformations populated by tau can be estimated using diffusion NMR spectroscopy. ${ }^{12}$ We estimated ensemble-averaged hydrodynamic radii of $56.8 \pm 0.2 \AA$ for wt tau and $60.2 \pm 0.3 \AA$ for the $\mathrm{E}$ mutant, both in $90 \% \mathrm{H}_{2} \mathrm{O} / 10 \% \mathrm{D}_{2} \mathrm{O}$ (Figure $\mathrm{S} 3$ ), supporting a less compact ensemble of conformers for the E-mutant. The value for wt tau is slightly larger than the hydrodynamic radius estimated previously in $100 \% \mathrm{D}_{2} \mathrm{O},{ }^{7}$ potentially as a result of different viscosities and stronger intramolecular hydrophobic interactions in $\mathrm{D}_{2} \mathrm{O} .{ }^{13}$

The raw PRE data do not constrain a single conformer but should be converted into ensembles of structures for which the PRE-derived distance restraints must be fulfilled by the complete ensemble. ${ }^{14}$ Previously, we calculated an ensemble of 10 conformers of wt tau that was in agreement with 2288 distance restraints derived from six MTSL positions. ${ }^{7}$ Here we calculated one ensemble for the wt protein and one for the E-mutant using 4646 and 4545 distance restraints, respectively. In addition, while in our previous study simulated annealing was used to drive the compaction of the ensemble under the influence of the PRE restraints, ${ }^{7}$ here subensembles of 200 structures were selected from a statistical coil ensemble of 30000 conformers using the program ASTEROIDS. ${ }^{14-16}$ The flexibility of the MTSL side chain was taken into account by rotamer modeling as described previously (Figure S4). In order to determine the optimal number of structures for the ensemble, complete PRE data sets for single MTSL positions were removed from the analysis, and these values were back-calculated from subensembles that were determined using the remaining 10 PRE data sets (Figures S5-S8). In addition, random samples of Gaussian noise were added to the input data to test the sensitivity of the ensembles to experimental uncertainties (Figures S9 and 10). To test the effect of local structural preferences on the properties of the ensemble, we performed two sets of test calculations: (i) local sampling for residues 395-425 with alternating 20\% more $\alpha$-helical and 20\% more $\beta$-sheet interspersed with standard sampling and (ii) $25 \%$ helix content for residues 428-437 in agreement with NMR chemical shifts ${ }^{7}$ (Figures S11 and S12).

The calculations showed that the mobility of the MTSL label, experimental uncertainties in the PREs, and local conformational preferences of the backbone do not affect the overall properties of the ensemble, although details in the ensembles might change. In addition, selection against the data resulted in slightly better reproduction than cross-validation. The contrary would be expected only if the system were overdetermined and the data that were left out were completely redundant. Thus, very large data sets are required for a full description of ensembles of disordered proteins. On the other hand, the cross-validation showed that the model, which used all of the data except that for one mutant, was still consistent with the data from this mutant. It also showed that subensembles of 200 structures describe the experimental data well and reproduce the back-calculated PREs with high quality (Figure 2 and Figure S13). Although each run of the program produces a different ensemble in terms of individual conformers, biophysical parameters such as long-range contacts and radii of gyration are constant.

The calculated ensembles support the interpretation of the raw data (Figure 2 and Figure S14): In the E-mutant, the N-terminal domain has a decreased probability to be in proximity to the proline-rich region, and the compaction of the $\mathrm{C}$-terminus is reduced. The overall dimensions of the ensemble of structures were slightly different, with hydrodynamic radius values of 58.9 and 60.0 Á for wt and E mutant tau, respectively (as predicted by HydroPRO ${ }^{17}$ ), which is in qualitative agreement with the experimental values. The structure calculations demonstrated that the experimental PRE profiles obtained for different MTSL positions are consistent. Importantly, we looked at differences between two data sets and therefore two ensembles with exactly the same extent and distribution of data.

Next, we asked whether changes in the global structure are connected to changes in the local structure of tau. To answer this, we assigned and measured ${ }^{15} \mathrm{~N}$ and ${ }^{13} \mathrm{C}^{\alpha}$ chemical shifts ${ }^{18}$ as well as ${ }^{3} J_{\text {HNHA }}$ scalar couplings in the E-mutant and compared them to those of wt tau. Both proteins showed only small deviations from random coil values of ${ }^{13} \mathrm{C}^{\alpha}$ chemical shifts, in agreement with their disordered nature (Figure S15). Direct comparison of the experimental ${ }^{13} \mathrm{C}^{\alpha}$ chemical shifts for wt and E-mutant tau demonstrated that local conformational changes induced by pseudophosphorylation are small and restricted to the vicinity of the mutation. Rigid secondary structure is not induced, in agreement with ${ }^{3} J_{\mathrm{HNHA}}$ scalar couplings (Figure S15) and previous work on short tau peptides. ${ }^{19}$ Despite the fact that the PHF1 epitope near the C-terminus contains only two phosphorylation sites (396 and 404), ${ }^{13} \mathrm{C}^{\alpha}$ chemical shift changes were more pronounced and affected a larger set of residues than did glutamic acid mutations in the proline-rich regions, suggesting that the PHF1 epitope is more prone to conformational changes.

In summary, we have determined an ensemble of conformers of 441-residue tau on the basis of a large number of distance restraints derived from paramagnetic relaxation enhancement and compared it to an equally well defined ensemble for a mutant protein mimicking phosphorylation. Our data demonstrate that pseudophosphorylation at the AT8, AT100, and PHF1 epitopes reduces the electrostatic attraction between the $\mathrm{N}$-terminal domain and the proline-rich region of tau and causes a weakening of the network of transient long-range interactions of 441-residue tau. Aggregation studies have previously suggested that the identical mutant protein aggregates faster than wt tau. ${ }^{9}$ Together with studies on the Parkinson-related protein $\alpha$-synuclein, in which long-range interactions between the C-terminal tail and the hydrophobic central part delay aggregation, ${ }^{20,21}$ our data indicate that networks of transient long-range interactions are common properties of intrinsically disordered proteins and important for aggregation.

\section{ASSOCIATED CONTENT}

Supporting Information. Eleven PRE broadening profiles, chemical shifts, and ${ }^{3} J$ couplings for wt and E-mutant tau. This material is available free of charge via the Internet at http://pubs.acs.org.

\section{AUTHOR INFORMATION}

\section{Corresponding Author}

mzwecks@gwdg.de 


\section{ACKNOWLEDGMENT}

We thank Ilka Lindner for excellent technical support, Eva-Maria Mandelkow and Christian Griesinger for discussions, and the Max Planck Society and the DFG (ZW 71/2-2 and 7-1 to M.Z.) and TAUSTRUCT - ANR MALZ 2010 (to M.B.) for financial support.

\section{REFERENCES}

(1) Ballatore, C.; Lee, V. M.; Trojanowski, J. Q. Nat. Rev. Neurosci. 2007, 8, 663-672.

(2) Garcia, M. L.; Cleveland, D. W. Curr. Opin. Cell Biol. 2001, 13, 41-48.

(3) Bancher, C.; Brunner, C.; Lassmann, H.; Budka, H.; Jellinger, K.; Wiche, G.; Seitelberger, F.; Grundke-Iqbal, I.; Iqbal, K.; Wisniewski, H. M. Brain Res. 1989, 477, 90-99.

(4) Mi, K.; Johnson, G. V. Curr. Alzheimer Res. 2006, 3, 449-463.

(5) Gong, C. X.; Liu, F.; Grundke-Iqbal, I.; Iqbal, K. J. Neural Transm. 2005, 112, 813-838.

(6) Cleveland, D. W.; Hwo, S. Y.; Kirschner, M. W. J. Mol. Biol. 1977, 116, 227-247.

(7) Mukrasch, M. D.; Bibow, S.; Korukottu, J.; Jeganathan, S.; Biernat, J.; Griesinger, C.; Mandelkow, E.; Zweckstetter, M. PLoS Biol. 2009, 7, No. e34.

(8) Dyson, H. J.; Wright, P. E. Chem. Rev. 2004, 104, 3607-3622.

(9) Jeganathan, S.; Hascher, A.; Chinnathambi, S.; Biernat, J.; Mandelkow, E. M.; Mandelkow, E. I. Biol. Chem. 2008, 283, 32066-32076.

(10) Sun, Q.; Gamblin, T. C. Biochemistry 2009, 48, 6002-6011.

(11) Gillespie, J. R.; Shortle, D. I.Mol. Biol. 1997, 268, 170-184.

(12) Wilkins, D. K.; Grimshaw, S. B.; Receveur, V.; Dobson, C. M.; Jones, J. A.; Smith, L. I. Biochemistry 1999, 38, 16424-16431.

(13) Cioni, P.; Strambini, G. B. Biophvs. I. 2002, 82, 3246-3253.

(14) Salmon, L.; Nodet, G.; Ozenne, V.; Yin, G.; Jensen, M. R.; Zweckstetter, M.; Blackledge, M. I. Am. Chem. Soc. 2010, 132, 8407-8418.

(15) Nodet, G.; Salmon, L.; Ozenne, V.; Meier, S.; Jensen, M. R.; Blackledge, M. I. Am. Chem. Soc. 2009, 131, 17908-17918.

(16) Bernado, P.; Blanchard, L.; Timmins, P.; Marion, D.; Ruigrok, R. W.; Blackledge, M. Proc. Natl. Acad. Sci. U.S.A. 2005, 102, 17002-17007.

(17) Garcia De La Torre, J.; Huertas, M. L.; Carrasco, B. Biophys. J. 2000, 78, 719-730.

(18) Wishart, D. S.; Sykes, B. D. Methods Enzvmol. 1994, 239, 363-392.

(19) Bielska, A. A.; Zondlo, N. I. Biochemistry 2006, 45, 5527-5537.

(20) Bertoncini, C. W.; Jung, Y. S.; Fernandez, C. O.; Hoyer, W.; Griesinger, C.; Jovin, T. M.; Zweckstetter, M. Proc. Natl. Acad. Sci. U.S.A. 2005, 102, 1430-1435.

(21) Hoyer, W.; Cherny, D.; Subramaniam, V.; Jovin, T. M. Biochemistry 2004, 43, 16233-16242. 